BNL --47070

DE92 007187

\title{
Vacuum Requirements for RHIC
}

\author{
M. J. Rhoades-Brown and M. Harrison
}

December 1991

\section{DISCLAIMER}

This report was prepared as an account of work sponsored by an agency of the United States Government. Neither the United States Government nor any agency thereof, nor any of their employees, makes any warranty, express or implied, or assumes, any legal liability or responsibility for the accuracy, completeness, or usefulness of any info mation, apparatus, product, or process disclosed, or represents that its use would not infringe privately owned rights. Reference herein to any specific commercial product, process, or service by trade name, trademark, manufacturer, or otherwise does not necessarily constitute or imply its endorsement, recommendation, or favoring by the United States Government or any agency thereof. The views and opinions of authors expressed herein do not necessarily state or reflect those of the United States Government or any agency thereof.

\section{R H I C P R O J E T}

\author{
Brookhaven National Laboratory \\ Associated Universities, Inc. \\ Upton, NY 11973
}

Under Contract No. DE-AC02-76CH00016 with the UNITED STATES DEPARTMENT OF ENERGY 


\title{
Vacuum Requirements for RHIC
}

\author{
M.J. Rhoades-Brown and M. Harrison
}

\section{Introduction}

In this note the lifetime due to inelastic scattering of beam and residual gas ions is calculated in units of pressure (Torr). In addition, the transverse emittance growth due to elastic scattering is expressed in units of pressure. The definition of inelastic scattering includes both capture of an electron from a residual gas ion and ceritral nuclear collisions betwcen beam ion and gas atom. Emittance growth via elastic scattering is a simple consequence of multiple Coulomb scattering.

It is important to note that in an accelerator only the density of residual gas atoms is relevant to the machine operation. The measure of this density is the vacuum gauge, where this gauge is calibrated in pressure units at some known temperature $T_{G}$. The vacuum unit or pressure is of course temperature dependent, and thus when quoting vacuum requirements for RHIC it is vital to state the temperature at which the pressure is computed. It might be necessary to scale any computed gas density to the pressure appropriate for the measurement with the vacuum gauge. Typically, the vacuum gauge operates at room temperature $\sim 300^{\circ} \mathrm{K}$. An explanation on how to rescale pressure as a function of temperature is given in the text.

This note assumes the residual gas density in the so-called warm section $\left(300^{\circ} \mathrm{K}\right)$ of RHIC to be composed of $90 \% \mathrm{H}_{2}, 5 \% \mathrm{CH}_{4}$ and $5 \% \mathrm{CO}$. The gas in the cold section $\left(5^{\circ} \mathrm{K}\right)$ is assumed to be $100 \%$ He. The beam ions are taken to be ${ }^{197} \mathrm{Au}^{79+}$.

\section{Variation of Pressure with Temperature}

Vacuum requirements in an accelerator can be confusing, for the units of pressure vary with temperature. Furthermore, most vacuum gauges respond to a density of gas $\rho_{G}$, where this density is expressed in pressure units $\mathrm{P}_{G}$ at temperature $\mathrm{T}_{G}$. Of course, both $\rho_{G}$ and $\mathrm{T}_{G}$ can be different from the beam pipe values. In this note, $\mathrm{T}_{W}, \rho_{W}$ and $\mathrm{P}_{W}$ 
correspond to thermodynamic variables in the warm section of RHIC, and $\mathrm{T}_{c}, \rho_{c}, \mathrm{P}_{c}$ to the cold section.

The only quantity of interest to machine performance is the density of gas ions inside the beam pipe. From the ideal gas law PV $=$ RT, the equivalent pressure $\mathrm{P}^{\prime}$ that this density produces at temperature $\mathrm{T}^{\prime}$ is simply

$$
P^{\prime}=\left(T^{\prime} / T\right) P
$$

However, if the gauge responds to a density $\rho_{G}$, and is calibrated at temperature $\mathrm{T}_{G}$, we need Knudsens relations for gas flow between two distinct volumes connected by a small tube, i.e.,

$$
\begin{gathered}
P=\left(T / T_{G}\right)^{1 / 2} P_{G} \\
\rho=\left(T_{G} / T\right)^{1 / 2} \rho G .
\end{gathered}
$$

Equating $\mathrm{T}^{\prime}$ with $\mathrm{T}_{G}$, these relations give,

$$
P^{\prime}=\left(T_{G} / T\right)^{1 / 2} P_{G}
$$

As a striking example, if the residual gas density is at $5^{\circ} \mathrm{K}$, and the vacuum gauge is at room temperature $\left(300^{\circ} \mathrm{K}\right)$, the equivalent pressure at $300^{\circ} \mathrm{K}$ is scaled by a factor of $300 / 5$ over the pressure at $5^{\circ} \mathrm{K}$. Moreover, this pressure is a factor of 7.74 higher than the pressure reading on the gauge.

\section{Inelastic Scattering Lifetimes}

The inelastic lifetime $\tau_{I}$ due to both electron capture and nuclear scattering is given by

$$
\frac{1}{\tau_{I}}=c \rho\left(\sigma_{\mathrm{C}}+\sigma_{\mathrm{N}}\right)
$$

where $\rho$ is the residual gas density (atoms $\mathrm{cm}^{-3}$ ), $\mathrm{c}$ is the velocity of light, $\sigma_{\mathrm{C}}$ is the capture cross section and $\sigma_{\mathrm{N}}$ is the nuclear collision cross section. Inserting Avogadro's number, 
$\mathrm{N}_{\mathrm{A}}=6.023 \times 10^{23}$ molecules $/$ mole, at a standard volume of 22.414 litres $/ \mathrm{moles}$, and a standard temperature $\mathrm{T}_{0}$ of $273.15^{\circ} \mathrm{K}$ gives in pressure units of Torr,

$$
\begin{gathered}
\frac{1}{\tau_{I}}=3.82 \times 10^{30} P_{o}(\text { Torr })\left(\sigma_{\mathrm{C}}\left(\mathrm{cm}^{2}\right)+\sigma_{\mathrm{N}}\left(\mathrm{cm}^{2}\right)\right) \text { hours }^{-1} \\
\left(T_{o}=273.15^{\circ} \mathrm{K}\right) \\
=3.48 \times 10^{30} P_{G}(\text { Torr })\left(\sigma_{\mathrm{C}}\left(\mathrm{cm}^{2}\right)+\sigma_{\mathrm{N}}\left(\mathrm{cm}^{2}\right)\right) \text { hours }^{-1} \\
(\text { WarmSection }) \\
=2.69 \times 10^{31} P_{G}(\text { Torr })\left(\sigma_{\mathrm{C}}\left(\mathrm{cm}^{2}\right)+\sigma_{\mathrm{N}}\left(\mathrm{cm}^{2}\right)\right) \text { hours }^{-1}
\end{gathered}
$$

(ColdSection)

Consider first the warm section of RHIC. The capture cross section $\sigma_{\mathrm{C}}$ is composed of three distinct mechanisms. Radiative electron capture is simply the inverse of the photoelectric effect. If $\mathrm{Z}_{P}$ and $\mathrm{Z}_{T}$ are the projectile and target atomic numbers $\sigma_{\mathrm{REC}}$ scales as

$$
\sigma_{\mathrm{REC}}=a Z_{P}^{5} Z_{T} / \gamma
$$

where $\gamma$ is the beam Lorentz parameter and $a$ is a constant that depends on the quantum dynamics of the capture process. Non-radiative electron capture, i.e., straight transfer without photon emission scales as

$$
\sigma_{\mathrm{NREC}}=b Z_{P}^{5} Z_{T}^{5} / \gamma
$$

where $\mathrm{b}$ is to be determined from quantum mechanical considerations.

In heavy ion colliders, the possibility of creating an electron-positron pair and subsequently capturing the electron results in the third capture mechanism called vacuum capture. This mechanism scales as

$$
\sigma_{\mathrm{VAC}}=d Z_{P}^{5} Z_{T}^{2} \ln \left(\gamma / \gamma_{\mathrm{o}}\right)
$$


where $d$ and $\gamma_{0}$ are constants to be determined from quantum mechanical calculations.

In Table I the constants a, b, d, $\gamma_{0}$ are tabulated for $\mathrm{H}, \mathrm{C}, \mathrm{O}$ target atoms ${ }^{1}$ and a ${ }^{197} A u^{79+}$ projectile.

TABLE 1

\begin{tabular}{|c|c|c|c|c|}
\hline $\begin{array}{c}\text { Residual Gas } \\
\text { Atom }\end{array}$ & $\mathrm{a}\left(\mathrm{cm}^{2}\right)$ & $\mathrm{b}\left(\mathrm{cm}^{2}\right)$ & $\mathrm{d}\left(\mathrm{cm}^{2}\right)$ & $\gamma_{0}$ \\
\hline $\mathrm{H}$ & $7.4 \times 10^{-34}$ & $8.4 \times 10^{-43}$ & $6.2 \times 10^{-37}$ & 7.91 \\
\hline $\mathrm{C}$ & $7.4 \times 10^{-34}$ & $4.19 \times 10^{-42}$ & $6.2 \times 10^{-37}$ & 7.91 \\
\hline $\mathrm{O}$ & $7.4 \times 10^{-34}$ & $4.60 \times 10^{-42}$ & $6.2 \times 10^{-37}$ & 7.91 \\
\hline
\end{tabular}

Table I. Constants a, b, d, $\gamma_{0}$ for ${ }^{197} A u^{79+}$ projectile.

Using Table I, equations (4) - (5), and the appropriate gas composition percentages, the effective capture cross section is $\sigma_{\mathrm{C}}=2.5 \times 10^{-25} \mathrm{~cm}^{2}$ at $\gamma=30$ and $\sigma_{\mathrm{C}}=1.1 \times$ $10^{-25} \mathrm{~cm}^{2}$ at $\gamma=100$.

For central nuclear collisions, a simple energy independent "billiard ball" model is assumed.

In this model $\sigma_{N}=\pi R_{N}^{2}$ where $R_{N}=1.2\left(A_{P}^{1 / 3}+A_{T}^{1 / 3}\right) \mathrm{fm}$.

With our gas composition for the warm section, it is calculated that $\sigma_{\mathrm{N}}=4.65 \times$ $10^{-24} \mathrm{~cm}^{2}$.

Comparing $\sigma_{\mathrm{N}}$ with $\sigma_{\mathrm{C}}$, it can be seen that the energy independent central nuclear collisions dominate over capture at RHIC energies. This is in stark contrast to Booster or AGS energies. From Eq. (2), we find for the warm section of RHIC,

$$
\tau_{I}=6.04 \times 10^{-2}\left[P_{W}(\mu \text { Torr })\right]^{-1} \text { hours } \quad\left(T_{W}=300^{\circ} \mathrm{K}\right)
$$

where we assume $\mathrm{T}_{G}=\mathrm{T}_{W}$.

The lifetime quoted in Eq. (9) is for a gas density averaged around the whole ring. However, allowing for a $12 \times 20 \mathrm{~m}$ warm section for the septum magnet at injection, and $12 \times 43 \mathrm{~m}$ for the insertion warm sections gives the warm fraction of RHIC circumference as .2. Equation (9) is then modified to read, 


$$
\tau_{I}=.3\left[P_{W}(\mu \text { Torr })\right]^{-1} \text { hours }\left({ }^{\prime} \Upsilon_{W}=300^{\circ} K\right) \quad .
$$

Hence for a pressure of $5 \times 10^{-10}$ Torr, we expect a beam lifetime of 600 hours in the warm section.

Consider now the cold section of RHIC. With $100 \%$ He gas we find $\sigma_{N}=2.48 \times 10^{-24} \mathrm{~cm}^{2}$. Taking into account the fraction of RHIC that is cold (.8), and ignoring contributions from capture we find

$$
\begin{aligned}
\tau_{I} & =2.42 \times 10^{-3}\left[P_{c}(\mu \text { Torr })\right]^{-1} \text { hours } & \left(T_{c}=5^{\circ} \mathrm{K}\right) \\
& =1.87 \times 10^{-2}\left[P_{G}(\mu \text { Torr })\right]^{-1} \text { hours } & \left(T_{G}=300^{\circ} \mathrm{K}\right)
\end{aligned}
$$

Hence for a pressure of $10^{-11}$ Torr, we expect a beam lifetime of 242 hours in the cold section.

Consider stated vacuum requirements in the $\mathrm{LHC}$ as an example. With their value for p- $\mathrm{H}_{2}$ scattering of $\sigma_{N}=10^{-29} \mathrm{~m}^{2}$ in Eq. (2), we get $P_{o}=1.091 \times 10^{-7}$ Torr at $273.15^{\circ} \mathrm{K}$. Using Eq. (1) to scale pressure with temperature gives their stated value of $1.2 \times 10^{-7}$ Torr at $293^{\circ} \mathrm{K}$.

\section{Emittance Growth Due to Elastic Scattering}

Multiple elastic Coulomb scattering will cause the transverse emittance of the beam to grow.

The rate of this growth is derived in Appendix A via the Fokker-Planck diffusion equation. The growth rate of the normalized emittance $\epsilon_{N}$ is

$$
\frac{d \epsilon_{N}}{d t}=\frac{\gamma \beta}{2} \dot{\Theta}_{r m s}^{2} \times F
$$

where

$$
\begin{gathered}
F=\frac{1}{2} \sum_{n} C_{n} D_{n}\left(\frac{-1}{\lambda_{n}^{2}}\right) e^{-\lambda_{n}^{2} \beta^{2} \dot{\Theta}_{r m a}^{2} t / 4 a^{2}}, \\
C_{n}=\frac{1}{J_{1}\left(\lambda_{n}\right)^{2}} \int_{o}^{1} f_{o}(z) J_{o}\left(\lambda_{n} \sqrt{z}\right) d Z,
\end{gathered}
$$




$$
D_{n}=\int_{0}^{\lambda n} d y y^{3} J_{0}(y)
$$

$\lambda_{n}$ is the root of Bessel function $\mathrm{J}_{0}\left(\lambda_{n}\right)=0$, and $\mathrm{a}$ is now defined to be the RHIC aperture. As explained in Appendix A, for an aperture that is large relative to a Gaussian beam parameter $\sigma, F \approx 1.0$. Hence

$$
\frac{d \epsilon_{N}}{d t} \simeq \frac{\gamma \beta}{2} \dot{\Theta}_{\mathrm{rms}}^{2}
$$

where $\beta$ is the average RHIC beta function, and $\dot{\Theta}_{r m s}^{2}$ is given by

$$
\dot{\Theta}_{r m s}^{2}=\left(\frac{15 M e V}{m_{p} c^{2} \gamma}\right)^{2} \frac{Z_{p}^{2}}{A_{P}^{2}} \frac{c}{L_{R A D}}
$$

where $m_{p}$ is the proton mass, and $L_{R A D}$ is the radiation length ${ }^{2}$ defined by,

$$
\frac{1}{L_{R A D}}=2 \alpha \frac{N_{A}}{A_{T}} Z_{T}^{2} r_{e}^{2} \rho \ln \left(R_{T} / R_{N}\right)
$$

where $\alpha=1 / 137, r_{e}=2.82 \times 10^{-13} \mathrm{~cm}$, and $R_{T}$ is the Thomas-Fermi screening radius,

$$
R_{T}=1.4(\hbar c) / \alpha Z_{T}^{1 / 3}{ } n_{e} c^{2}(c m)
$$

Expressing $\rho$ in terms of pressure, the effective radiation length $L_{\mathrm{RAD}}^{\mathrm{EFF}}$ for our warm section gas composition is calculated to be

$$
\frac{1}{L_{\mathrm{RAD}}^{\mathrm{EFF}}}=10^{-9} P_{W}(\text { Torr }) \mathrm{cm}^{-1} \quad\left(T_{W}=300^{\circ} \mathrm{K}\right)
$$

Hence for ${ }^{197} A u^{79+}$ ions it is found

$$
\begin{gathered}
\frac{d \epsilon_{N}}{d t}=112.5 \frac{P_{W}(\mu \text { Torr })}{\gamma} \mathrm{mm} \mathrm{mrad} \mathrm{hour}{ }^{-1} \\
\left(T_{W}=300^{\circ} \mathrm{K}\right)
\end{gathered}
$$

Allowing for the fraction of the ring taken up by the warm section gives 


$$
\begin{aligned}
& \frac{d \epsilon_{N}}{d t}=22.5 \frac{P_{G}(\mu \text { Torr })}{\gamma} \mathrm{mm} \mathrm{mrad} \mathrm{hour}{ }^{-1} \\
& \left(T_{G}=300^{\circ} \mathrm{K}\right)
\end{aligned}
$$

Hence for $\mathrm{Au}$ beams at $\gamma=100$, the normalized emittance grows at a rate of $1.13 \times 10^{-4}$ $\mathrm{mm} \mathrm{mrad} \mathrm{hour}{ }^{-1}$ for a pressure of $5 \times 10^{-10}$ Torr in the warm section.

For the cold section of RHIC,

$$
\frac{1}{L_{\mathrm{RAD}}^{\mathrm{EFF}}}=2.13 \times 10^{-8} P_{o}(\text { Torr }) \mathrm{cm}^{-1} \quad\left(T_{o}=5^{\circ} \mathrm{K}\right)
$$

Hence for ${ }^{197} A u^{79+}$ ions,

$$
\begin{gathered}
\frac{d \epsilon_{N}}{d t}=2.40 \times 10^{3} \frac{P_{c}(\mu \text { Torr })}{\gamma} \mathrm{mm} \mathrm{mrad} \mathrm{hour}{ }^{-1} \\
\left(T_{c}=5^{\circ} \mathrm{K}\right) \\
=3.11 \times 10^{2} \frac{P_{G}(\mu \mathrm{Torr})}{\gamma} \mathrm{mm} \mathrm{mrad} \mathrm{hour}{ }^{-1} \\
\left(T_{G}=300^{\circ} \mathrm{K}\right)
\end{gathered}
$$

Hence for $\mathrm{Au}$ beams of $\gamma=100$, the normalized emittance grows at a rate of $2.4 \times 10^{-4}$ $\mathrm{mm}$ mrad hour ${ }^{-1}$ for a pressure of $10^{-11}$ Torr in the cold section.

\section{References}

1) R. Anholt and V. Becker, Phys. Rev. $\underline{\mathrm{A} 36}, 4628$ (1987).

2) Y.S. Tsai, Rev. Mod. Phys. $\underline{46}, 815$ (1974). 


\section{Appendix A}

Consider emittance growth via the Fokker-Planck equation,

$$
\frac{\partial f}{\partial t}=D \frac{\partial}{\partial W}\left(W \frac{\partial f}{\partial W}\right)
$$

where $f$ is the time-dependent distribution of beam particles in the transverse plane. If $\tau$ $=\mathrm{Dt} / \mathrm{W}_{a}$ and $\mathrm{Z}=\mathrm{W} / \mathrm{W}_{a}$ where $\mathrm{D}$ is the diffusion constant and $\mathrm{W}_{a}=\mathrm{a}^{2} / \beta$, where $\mathrm{a}$ is the aperture and $\beta$ the beta function,

$$
\frac{\partial f}{\partial \tau}=\frac{\partial}{\partial Z}\left(Z \frac{\partial f}{\partial Z}\right)
$$

However if $f(Z, \tau=0) \equiv f_{o}(Z)$ then the standard solution to Eq.(A.1) is

$$
f(Z, \tau)=\sum_{n} C_{n} J_{0}\left(\lambda_{n} \sqrt{Z}\right) e^{-\lambda_{n}^{2} \tau / 4}
$$

where

$$
C_{n}=\frac{1}{J_{1}\left(\lambda_{n}\right)^{2}} \int_{0}^{1} f_{0}(Z) J_{0}\left(\lambda_{n} \sqrt{Z}\right) d Z
$$

and $\mathrm{J}_{o}\left(\lambda_{n}\right)=0$

We assume a Gaussian for $f_{o}(Z)$,

$$
\begin{gathered}
f_{o} d Z=\frac{1}{2 \pi \sigma_{0}^{2}} e^{-r^{2} / 2 \sigma_{o}^{2}} r d r d \theta=\frac{a^{2}}{2 \sigma_{o}^{2}} e^{-a^{2} Z / 2 \sigma_{o}^{2}} d Z, \\
(r=a \sqrt{Z})
\end{gathered}
$$

and are interested in how $\sigma$ changes in time. Looking at the second moment $\mathrm{I}(\tau=0)$ of $f_{0}(Z)$ gives

$$
I(\tau=0)=2 \sigma_{o}^{2}-2 \sigma_{o}^{2} e^{-\alpha}[\alpha+1]
$$




$$
\frac{d \epsilon_{N}}{d t}=\frac{\gamma \beta \dot{\Theta}_{r m s}^{2}}{2} \sum_{n} \frac{1}{2} C_{n} D_{n}\left(\frac{-1}{\lambda_{n}^{2}}\right) e^{-\lambda_{n}^{2} \beta^{2} \dot{\theta}_{r m s}^{2} / 4 a^{2}}
$$

Evaluating the sum over $\mathrm{n}$ for large a gives a value equal to approximately unity (1.0019 for $\alpha=10$ and 1.0741 for $\alpha=30)$ when $\alpha$ is large $(\geq 5)$.

Hence for large aperture,

$$
\frac{d \epsilon_{N}}{d t} \simeq \frac{\gamma \beta \dot{\Theta}_{r m s}^{2}}{2}
$$


where $\alpha=a^{2} / 2 \sigma_{0}^{2}$. Hence as $\alpha \rightarrow \infty \mathrm{I}(\tau=0)=2 \sigma_{o}^{2}$ as required. Similarly, looking at the second moment $I(\tau)$ of $f(Z, \tau)$ gives,

$$
I(\tau)=2 \sigma_{0}^{2} G(\tau)
$$

where

$$
G(\tau)=2 \alpha \sum_{n} C_{n} D_{n} \frac{1}{\lambda_{n}^{4}} e^{-\lambda_{n}^{2} \tau / 4}
$$

and

$$
D_{n}=\int_{0}^{\lambda_{n}} d y y^{3} J_{0}(y)
$$

These equations show how the Gaussian spreads as a function of the universal parameter $\tau$, where $\tau$ is proportional to the diffusion constant.

Let us rewrite Eqs. (A.6) - (A.8) for emittance growth in real time. The diffusion constant for our problem is

$$
D=\beta \dot{\Theta}_{r m s}^{2}
$$

hence

$$
\tau=\frac{a^{2}}{\beta^{2}} \frac{1}{\dot{\Theta}_{r m s}^{2}} \tau
$$

Thus, from Eq. (A.6)

$$
\begin{gathered}
\frac{d I}{d t}=2 \sigma_{o}^{2} \frac{d \tau}{d t} \frac{d G(\tau)}{d \tau} \\
=\frac{\beta^{2} \dot{\Theta}_{r m s}^{2}}{2} \sum_{n} C_{n} D_{n}\left(\frac{-1}{\lambda_{n}^{2}}\right) e^{-\lambda_{n}^{2} \beta^{2} \dot{\Theta}_{r m}^{2}, t / 4 a^{2}}
\end{gathered}
$$

Using $\sigma(t)=\sqrt{\epsilon_{N} \beta / \gamma}$ and $\mathrm{I}=2 \sigma^{2}(\mathrm{t})$ gives 

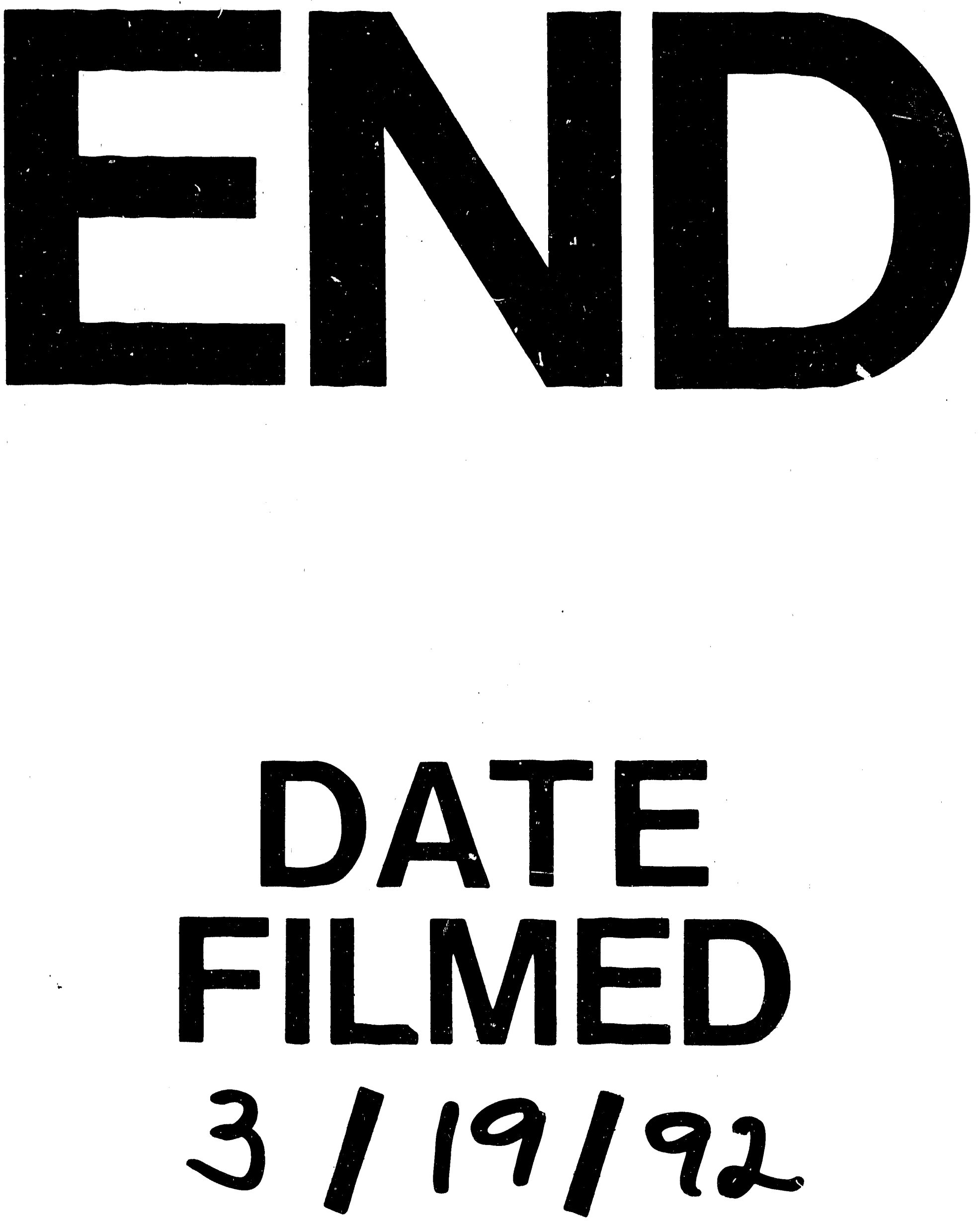